\title{
Nanodroplets on rough hydrophilic and hydrophobic surfaces
}

\author{
C. Yang, ${ }^{1}$ U. Tartaglino, ${ }^{1}$ and B.N.J. Persson ${ }^{1}$ \\ ${ }^{1}$ Institut für Festkörperforschung, Forschungszentrum Jülich, D-52425 Jülich, Germany
}

\begin{abstract}
We present results of Molecular Dynamics (MD) calculations on the behavior of liquid nanodroplets on rough hydrophobic and hydrophilic solid surfaces. On hydrophobic surfaces, the contact angle for nanodroplets depends strongly on the root mean square roughness amplitude, but it is nearly independent of the fractal dimension of the surface. Since increasing the fractal dimension increases the short-wavelength roughness, while the long-wavelength roughness is almost unchanged, we conclude that for hydrophobic interactions the short-wavelength (atomistic) roughness is not very important. We show that the nanodroplet is in a Cassie-like state. For rough hydrophobic surfaces, there is no contact angle hysteresis due to strong thermal fluctuations, which occur at the liquidsolid interface on the nanoscale. On hydrophilic surfaces, however, there is strong contact angle hysteresis due to higher energy barrier. These findings may be very important for the development of artificially biomimetic superhydrophobic surfaces.
\end{abstract}

Reference: Eur. Phys. J. E 25, 139-152 (2008)

DOI: $10.1140 /$ epje/i2007-10271-7

Preprint arXiv:0710.3264

\section{INTRODUCTION}

In the year of 1805, Thomas Young and Pierre Simon de Laplace proposed that an interface between two materials has specific energy, the so-called interfacial energy, which is proportional to the interfacial surface area 1, 2, 3]. This concept is the basis for the field of wetting, which has become an extremely hot topic in the last two decades [4, 5], thanks to biological and high-tech applications, ranging from self-cleaning surfaces, microelectronics and thin film coatings, to image formation that involve the spreading of liquids on solid surfaces.

Wetting describes the contact between a fluid and a solid surface. Liquids with high surface tension (usually reflecting strong intra-molecular bonds), or liquids on low-energy solid surfaces, usually form nearly (complete) spherical droplets, whereas liquids with low surface tension, or liquids on high-energy surfaces, usually spread out on (or wet) the surfaces. This phenomenon is a result of minimization of interfacial energy. Thus, if a surface has a high free energy, most liquids will spread on the surface since this will usually lower the free energy.

Wetting phenomena have been widely studied both theoretically [6, 7] and experimentally [8, 9] in connection with the physics of surfaces and interfaces. The behavior of liquids on smooth solid surfaces is rather well understood. However, for rough solid surfaces the situation is much less clear, even though roughness occurs on practically all real surfaces of engineering or biological interest. Studies (and classification) of disordered and inhomogeneous surfaces [10] should have significant impact on the problem of liquid contact angle and wetting of rough substrates 11, 12, 13, 14, 15]

The fascinating water repellents of many biological surfaces, in particular plant leaves, have recently attracted great interest for fundamental research as well as practical applications 16, 17, 18, 19, 20, 21, 22, 23]. The ability of these surfaces to eliminate water beads completely

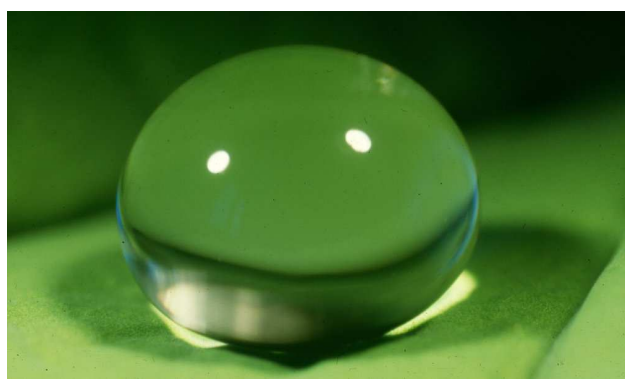

FIG. 1: A droplet on a superhydrophobic surface: The droplet touch the leaf only at a few points and forms a ball. It completely rolls off at the slightest declination. Adapted from Ref. [16] with permission.

and thereby wash off contamination very effectively has been termed the Lotus effect, although it is observed not only on the leaves of the Lotus plant (Fig. 1), but also on many other plants such as strawberry, raspberry and so on. Water repellents are very important in many industrial and biological processes, such as prevention of the adhesion of snow, rain drops and fog to antennas, self-cleaning windows and traffic indicators, low-friction surfaces and cell mobility 24, 25, 26].

Most leaves that exhibit strong hydrophobicity have hierarchical surface roughness with micro- and nanostructures made of unwettable wax crystals, which maximize the contact angle with water and most other liquids. Fig. 2 shows epidermal cells (microscale roughness) covered with wax crystals (nanoscale roughness). The wax crystals exhibit a relative high contact angle with water, which is enhanced by the surface roughness. Water droplets on the rough wax surface tend to minimize the contact between the surface and the droplet by forming nearly spherical droplets, as approximately described by the two classical models due to Wenzel[27] and Cassie 28] 


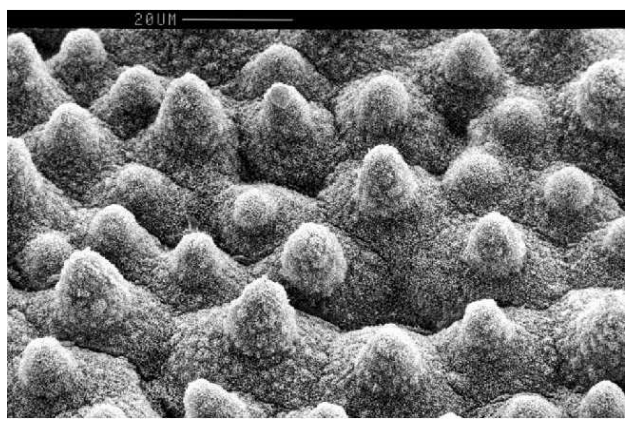

FIG. 2: A leaf surface with roughness on several length scales optimized via nature selection for hydrophobicity and selfcleaning. Through the combination of microstructure (cells) and nanostructure (wax crystals) the macroscopic water contact angle $\theta_{0}$ is maximized. Adapted from Ref. [16] with permission.

(see below). As a result the leaves have also a selfcleaning property: because of the small adhesion energy (and small contact area) between contamination particles and the rough leaf[17], during raining water drops roll away removing the contamination particles from the leaf surface.

The hydrophobicity of solid surfaces is determined by both the chemical composition and the geometrical micro- or nanostructure of the surface 8, 29, 30]. Understanding the wetting of corrugated and porous surfaces is a problem of long standing interest in areas ranging from textile science 31] to catalytic reaction engineering [32]. Renewed interest in this problem has been generated by the discoveries of surfaces with small scale corrugations that exhibit very large contact angles for water and other liquids - in some cases the contact angle is close to $180^{\circ}$. Such surfaces are referred to as superhydrophobic 33.

In this paper we present results of Molecular Dynamics (MD) calculations on the behavior of liquid nanodroplets on rough hydrophilic and hydrophobic solid surfaces. We find that for hydrophobic surfaces, the contact angle for nanodroplets depends strongly on the root mean square surface roughness amplitude, but is nearly independent of the fractal dimension $D_{\mathrm{f}}$ of the surface. For hydrophobic rough surfaces we do not detect any contact angle hysteresis. Both results can be explained by the strong thermal fluctuations which occur at the liquid-solid interface on the nanoscale. On hydrophilic surfaces, however, strong contact angle hysteresis has been found due to the higher energy barrier for interfacial liquid density fluctuations. These findings may be crucial for the development of artificial biomimetic superhydrophobic surfaces.

\section{THEORETICAL BACKGROUND}

In this section we briefly describe some results from the theory of the liquid-solid contact angle, which are necessary for the interpretation of the numerical results

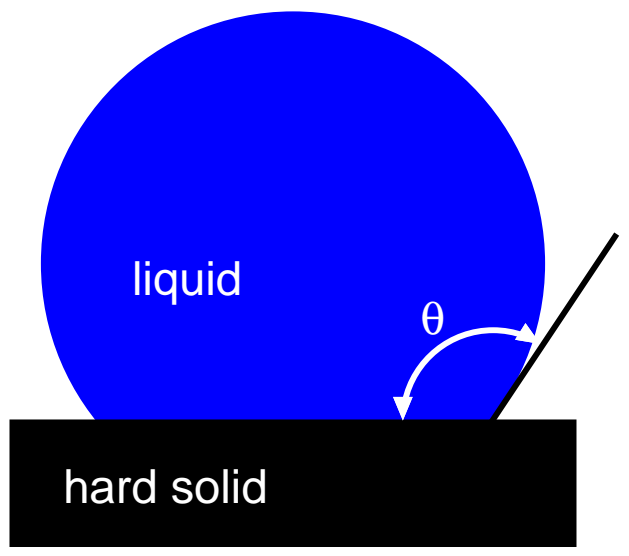

FIG. 3: Liquid droplet on flat substrate. The contact angle $\theta$ is between 0 (complete wetting) and $\pi$.

presented in Sec. IV] We emphasize the importance of thermal fluctuations for the contact dynamics at the nanoscale as compared to micrometer or macroscopic dimensions.

\section{A. Flat surfaces}

If gravitational effects can be neglected, a liquid droplet on a flat substrate forms a spherical cap, see Fig. 3. The contact angle $\theta$ is determined by the minimization of the free energy and depends on the interfacial free energies per unit area: solid/liquid $\gamma_{\mathrm{sl}}$, solid/vapor $\gamma_{\mathrm{sv}}$ and liquid/vapor $\gamma_{\mathrm{lv}}$. Minimizing of the surface free energy, with the constrain of fixed volume of the droplet, gives the Young's equation, first proposed by Thomas Young about two hundred years ago:

$$
\gamma_{\mathrm{sl}}+\gamma_{\mathrm{lv}} \cos \theta=\gamma_{\mathrm{sv}}
$$

Complete wetting corresponds to $\theta=0$, and typically happens for liquids with low surface tension $\gamma_{\mathrm{lv}}$, and on solids with high surface energy $\gamma_{\mathrm{sv}}$. Liquids with high surface tension on surfaces with low surface energy tend to form droplets with high contact angle $\theta$. Eq. (1) was deduced for a substrate which is assumed to be perfectly smooth, homogeneous, and rigid. However, in reality, structured or rough surfaces are quite common. So it's necessary to know how the contact angle behaves on rough surfaces.

\section{B. Rough surfaces: minimum free energy state}

Most surfaces of practical interest have roughness on many different length scales. For simple periodic surface profiles one may develop accurate analytical treatments of the liquid droplet contact angle (see e.g., Ref. [34]), 


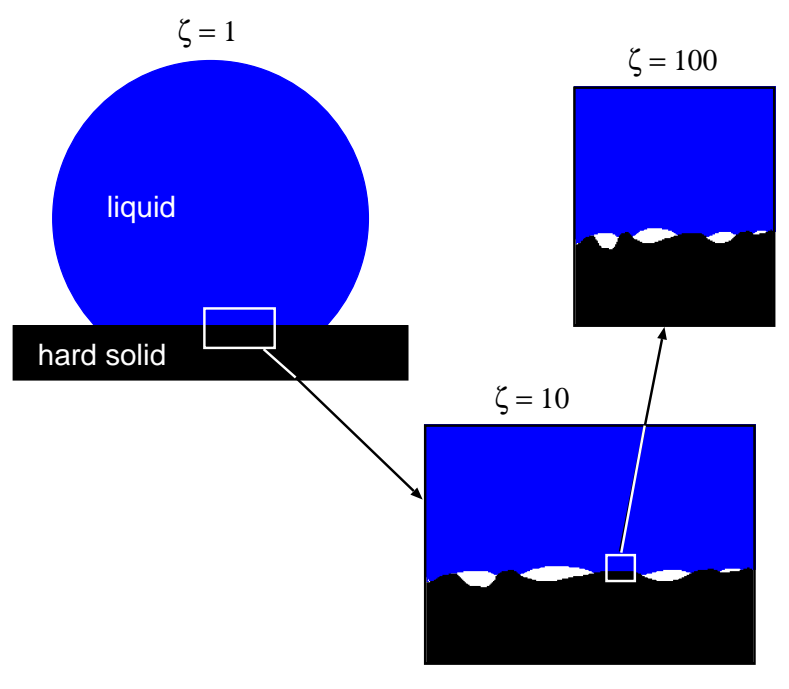

FIG. 4: Liquid droplet on a rough substrate. At the lowest magnification $\zeta$ the surface appears flat and the liquid contact angle is $\theta_{0}$. At increasing magnification surface roughness is observed and the liquid will in general only make contact with the substrate in some asperity contact regions.

but for randomly rough surfaces the situation is much more complex. For surfaces with random roughness, e.g., self-affine fractal surfaces (see below), one may develop a general theory based on the study of the system at

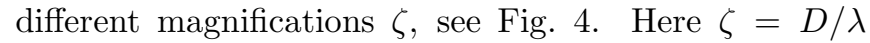
where $D$ is the diameter of the droplet-substrate (apparent) contact area and $\lambda$ the resolution. One can introduce effective interfacial liquid-solid and solid-vapor free energies (per unit area) $\gamma_{\mathrm{sl}}(\zeta)$ and $\gamma_{\mathrm{sv}}(\zeta)$ which depend on the magnification $\zeta[35]$. At the highest magnification $\zeta_{1}$, corresponding to nanometer (or atomistic) resolution, these quantities reduce to those for the flat surface [36], $\gamma_{\mathrm{sl}}\left(\zeta_{1}\right)=\gamma_{\mathrm{sl}}$ and $\gamma_{\mathrm{sv}}\left(\zeta_{1}\right)=\gamma_{\mathrm{sv}}$. Since the substrate appears flat at the lowest magnification $\zeta=1$, the macroscopic contact angle (corresponding to $\zeta=1$ ) is obtained using the Young's equation with $\gamma_{\mathrm{sl}}$ and $\gamma_{\mathrm{sv}}$ replaced by $\gamma_{\mathrm{sl}}(1)$ and $\gamma_{\mathrm{sv}}(1)$, i.e.

$$
\gamma_{\mathrm{sl}}(1)+\gamma_{\mathrm{lv}} \cos \theta_{0}=\gamma_{\mathrm{sv}}(1)
$$

The change in the surface free energy (per unit area) when a liquid with a flat surface is brought in contact with the substrate is

$$
\Delta F / A_{0}=\gamma_{\mathrm{sl}}(1)-\gamma_{\mathrm{sv}}(1)-\gamma_{\mathrm{lv}}=-\gamma_{\mathrm{lv}}\left(1+\cos \theta_{0}\right)
$$

where $A_{0}$ is the (projected) surface area. Note that increasing contact angle $\theta_{0}$ corresponds to a increasing interfacial free energy. Thus, if a liquid drop can occur in several metastable states on a surface, the state with the smallest contact angle corresponds to the (stable) minimal free-energy state.

Using Eq. (2) it is trivial to derive the results of the so called Wenzel[27] and Cassie 28] models. In the Wenzel model it is assumed that complete contact occurs at the liquid-solid interface. Thus

$$
\gamma_{\mathrm{sv}}(1)=r \gamma_{\mathrm{sv}}\left(\zeta_{1}\right), \quad \gamma_{\mathrm{sl}}(1)=r \gamma_{\mathrm{sl}}\left(\zeta_{1}\right),
$$

where $r=A / A_{0}>0$ is the ratio between the surface area $A$ of the rough substrate, and the projected (or nominal) surface area $A_{0}$. Substituting (3) into (2) gives the contact angle $\theta_{0}$ on the rough surface in terms of the contact angle $\theta$ on the microscopically flat surface of the same material (Wenzel equation):

$$
\cos \theta_{0}=r \cos \theta .
$$

In the Cassie model [28] it is assumed that some air (or vapor) remains trapped between the drop and the cavities of the rough surface. In this case the interface free energy

$$
\begin{gathered}
\gamma_{\mathrm{sv}}(1)=r \gamma_{\mathrm{sv}}\left(\zeta_{1}\right), \\
\gamma_{\mathrm{sl}}(1)=\phi r \gamma_{\mathrm{sl}}\left(\zeta_{1}\right)+(1-\phi)\left(r \gamma_{\mathrm{sv}}\left(\zeta_{1}\right)+\gamma_{\mathrm{lv}}\right),
\end{gathered}
$$

where $\phi$ is the fraction of the (projected) area where the liquid is in contact with the solid. Substituting (5) and (6) in (2) gives

$$
\cos \theta_{0}=r \cos \theta-(1-\phi)(1+r \cos \theta) .
$$

Note that for $\phi=1$, (7) reduces to (4). In the original Cassie model it was assumed that $r=1$. We note that while the Wenzel theory is exact if the liquid is in contact with the substrate everywhere within the nominal liquid-substrate contact area, the Cassie theory is always approximate and often not very accurate. This is easily understood from Fig. [5 which shows the interface between a liquid and a solid. $\phi<1$ is the ratio between the projected liquid-solid contact area and the nominal (or apparent) contact area $A_{0}$. Because the solid surface is curved, the actual liquid-solid contact area will be $A_{0} \phi s$ where $s>1$. Analogously, since in general the liquidvapor interface is curved (in spite of the fact that the total curvature $1 / R_{1}+1 / R_{2}$ may vanish) and tilted (relative to the average surface plane), the total liquid-vapor interface area is $A_{0}(1-\phi) s^{\prime}$, with $s^{\prime}>1$. Similarly, the solid-vapor interface area equals $A_{0}(1-\phi) s^{\prime \prime}$ with $s^{\prime \prime}>1$. In deriving (7) it is assumed that $s=s^{\prime \prime}=r$ and $s^{\prime}=1$.

Of the two states, Cassie and Wenzel, the stable one, that is the one with lower free energy, is the one with larger $\cos \theta_{0}$. Comparing (4) and (7) shows that the value of $\cos \theta_{0}$ for the Cassie state is larger if $1+r \cos \theta<0$ or

$$
\cos \theta<-1 / r .
$$

Since $r$ is a measure of the magnitude of the surface roughness, we may qualitatively state that only for hydrophobic surfaces (with $\cos \theta<0$ or $\theta>90^{\circ}$ ) with large enough roughness (i.e., large enough $r=A / A_{0}$ ) will the Cassie state be the thermodynamically stable state. 


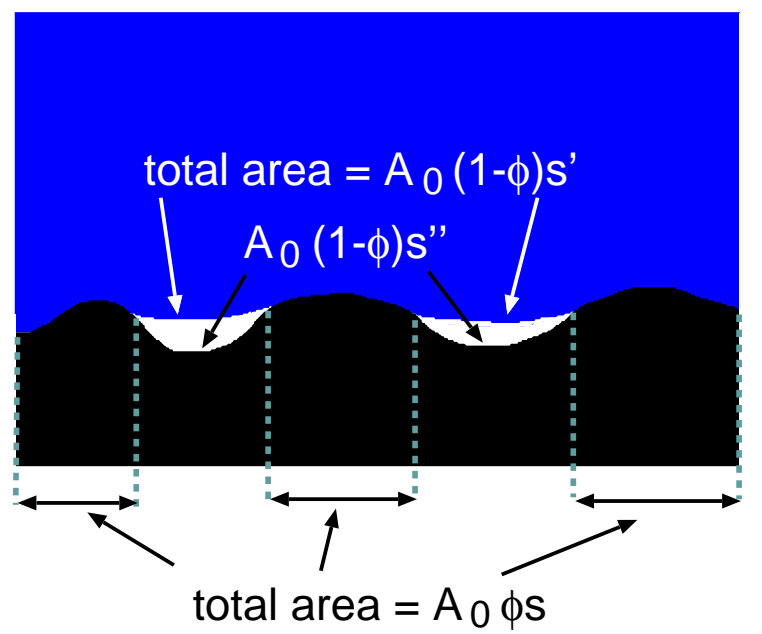

FIG. 5: The interface between liquid and solid. $\phi<1$ is the ratio between the projected liquid-solid contact area and the nominal (or apparent) contact area $A_{0}$. Because the solid surface is curved, the actual liquid-solid contact area will be $A_{0} \phi s$ where $s>1$. Similarly, since in general the liquidvapor interface is curved (in spite of the fact that the total curvature $1 / R_{1}+1 / R_{2}$ may vanish) and tilted (relative to the average surface plane) the total liquid-vapor interface area is $A_{0}(1-\phi) s^{\prime}$, with $s^{\prime}>1$. Similarly, the solid-vapor interface area equals $A_{0}(1-\phi) s^{\prime \prime}$ with $s^{\prime \prime}>1$.

The approach described above, where the interface is studied at different magnifications, is very general and a similar approach has recently been developed for the contact mechanics between elastic solids with randomly rough surfaces [37] (see also Ref. [38]).

It is well known that the roughness of a hydrophobic solid (with $\theta>90^{\circ}$ on the flat substrate) enhances its hydrophobicity. If the contact angle of water on such flat solids is of the order of $100^{\circ}$ to $120^{\circ}$, on a rough or microtextured surface it may be as high as $150^{\circ}$ to $175^{\circ}[26,38,39]$. Both the Wenzel model and the Cassie model can explain this effect.

Let us consider the simplest surface roughness consisting of a periodic rectangular roughness profile as illustrated in Fig. 6. (a) (xz-plane). The free energy (per unit surface area) for the Cassie state shown in the figure is

$$
\gamma_{\mathrm{C}}=\left[(a+2 h) \gamma_{\mathrm{sv}}+a \gamma_{\mathrm{lv}}+b \gamma_{\mathrm{sl}}\right] /(a+b)
$$

The free energy for the Wenzel state (complete contact) is

$$
\gamma_{\mathrm{W}}=(a+b+2 h) \gamma_{\mathrm{sl}} /(a+b)
$$

Using (1) we can write the difference in free energy

$$
\gamma_{\mathrm{C}}-\gamma_{\mathrm{W}}=\gamma_{\mathrm{lv}}[a(1+\cos \theta)+2 h \cos \theta] /(a+b)
$$

Thus, the Cassie state has a lower free energy than the Wenzel state if

$$
\cos \theta<-\left(1+\frac{2 h}{a}\right)^{-1}
$$

(a)

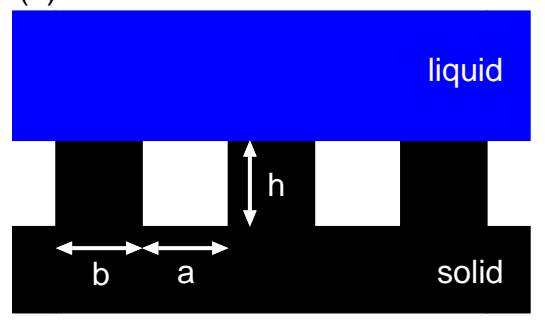

(b)

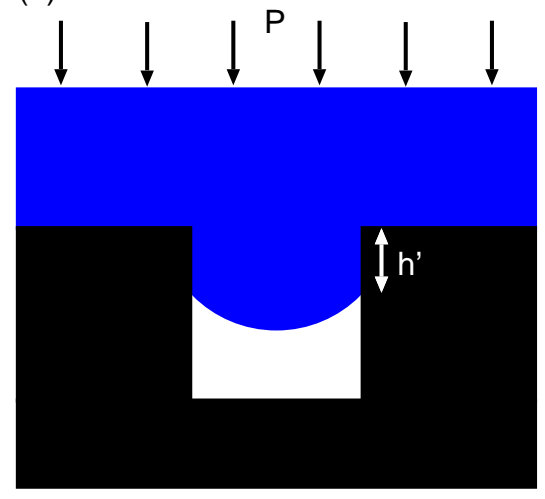

FIG. 6: (a) Liquid drop (in the Cassie state) in contact with a surface with periodic surface roughness. (b) Even if the Cassie state (incomplete liquid-solid contact) is the ground state, with an applied pressure $p$ one can squeeze the droplet into the Wenzel state.

which is satisfied only if for the flat surface $\theta>90^{\circ}$, and if the ratio $h / a$ is large enough. Note that in this case $r=A / A_{0}=(a+b+2 h) /(a+b)$ so the (approximate) criteria (8) reduces to

$$
\cos \theta<-\left(1+\frac{2 h}{a+b}\right)^{-1}
$$

which is of similar general form as (9). In Nature strongly hydrophobic surfaces are often obtained by covering the surface with thin, long (so that $h / a \gg 1$ ) hydrophobic fibers. Thus, insects which move on top of water, e.g., water spiders (see Fig. 77) have a high density of thin wax coated hair on their legs. In addition, the hair fibers have nanoscale roughness which traps air and enhances the hydrophobicity [40]. In this case the water-leg contact will be in the Cassie state even when the insect is squeezed towards the water by the weight of the insect.

\section{Rough surfaces: activation barriers and hysteresis}

Consider a cylindrical cavity as in Fig. 6 (b) and assume first the Cassie state as in the figure. Let us apply a pressure $p$ to the droplet. In this case the liquid will 


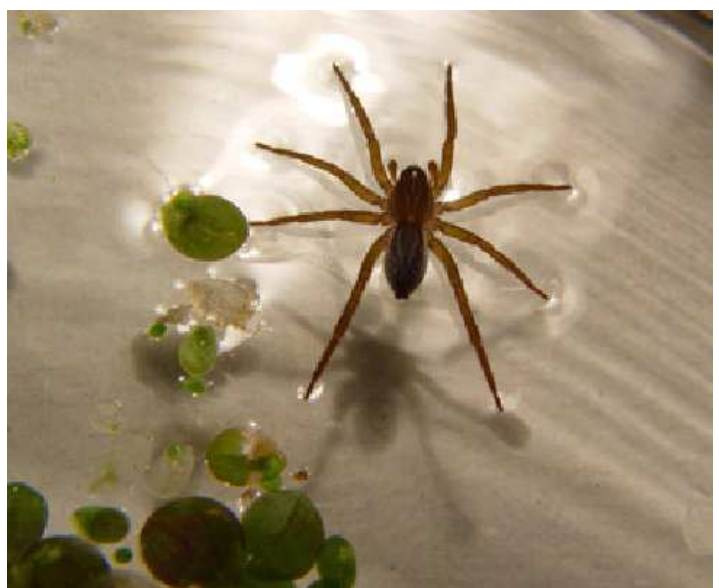

FIG. 7: Water spiders have thin hydrophobic (wax coated) hair with nanoscale roughness which trap air and enhance hydrophobicity.

bend inwards in the cavity and if the applied pressure is larger than a critical value $p_{c}$, the liquid will be squeezed into the cavity (we assume that the air in the cavity can leave the cavity, e.g., diffuse into the liquid). It is easy to show that the pressure

$$
p_{c}=-2 \gamma_{\mathrm{lv}} \cos \theta / R
$$

where $R$ is the radius of the cavity. To prove this relation, note that the pressure work to squeeze the liquid a distance $h^{\prime}$ into the cavity (see Fig. 6) is given by $p_{c} \pi R^{2} h^{\prime}$ and this must equal the change in interfacial free energy which equals $2 \pi R h^{\prime}\left(\gamma_{\mathrm{sl}}-\gamma_{\mathrm{sv}}\right)$. Using these equations and (11) gives (10).

From (10) it follows that if $\theta<90^{\circ}$ (hydrophilic interaction), $p_{c}<0$ and the liquid will be spontaneously sucked into the cavity and will fill out the cavity. If $\theta>90^{\circ}$ (hydrophobic interaction), $p_{c}>0$ and for nanometer sized cavities, the pressure $p_{c} \sim 100 \mathrm{MPa}$, so very high pressures are necessary for squeezing the liquid into narrow cavities. However, if the liquid is squeezed into the cavity and completely fills the cavity, then the resulting Wenzel state is (at least) metastable. However, for nanometer sized cavities thermal fluctuations may give rise to strong local fluctuations between the Cassie (empty cavity) and Wenzel (filled cavity) states. This is easy to understand since the energetic barrier (for a hydrophobic system) for going from the Cassie state to the Wenzel state will be of order $\varepsilon \sim p_{c} \pi R^{2} h=$ $-2 \pi R h \gamma_{\mathrm{lv}} \cos \theta$, and strong fluctuations on macroscopic time scales will occur as long as $\varepsilon \approx 0.7 \mathrm{eV}$ or less, and strong fluctuations on the nanosecond time scale occur if $\varepsilon \approx 0.4 \mathrm{eV}$ or less [note: the rate to jump over a barrier of height $\varepsilon$ is $w=\nu \exp \left(-\varepsilon / k_{\mathrm{B}} T\right)$ where typically $\nu \approx 10^{12} \mathrm{~s}^{-1}$; at room temperature $w \approx 1 \mathrm{~s}^{-1}$ if $\varepsilon \approx 0.7 \mathrm{eV}$ and $w \approx 10^{9} \mathrm{~s}^{-1}$ if $\left.\varepsilon \approx 0.4 \mathrm{eV}\right]$. In a typical case this condition is satisfied as long as $R$ and $h$ are of order of one nanometer or less. In our computer

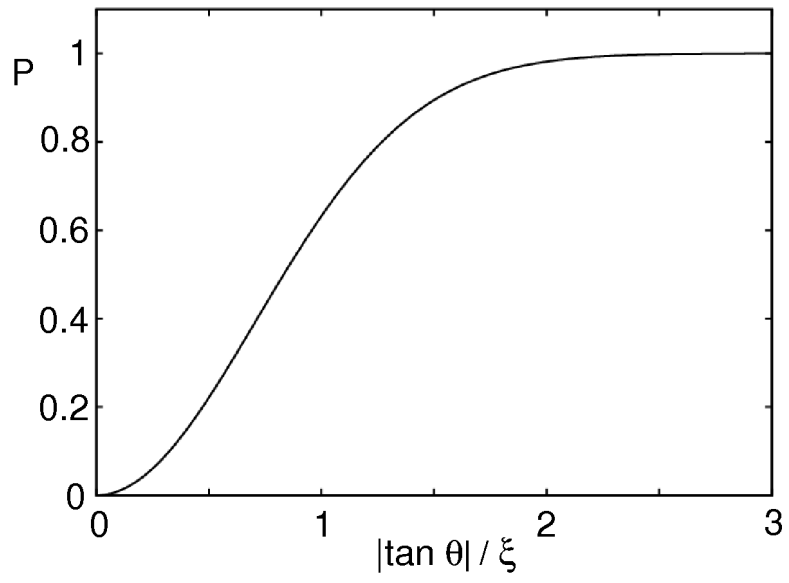

FIG. 8: The fraction $P$ of the surface area where the absolute value of the slope is smaller than $|\tan \theta|$ as a function of $|\tan \theta| / \xi$. See text for details.

simulations we do indeed observe very strong thermal fluctuations at the liquid-solid interface, in particular for rough hydrophobic surfaces, see Sec. IV B1

The Wenzel droplets are highly pinned, and the transition from the Cassie to the Wenzel state results in the loss of the anti-adhesive properties generally associated with superhydrophobicity. However, for nanodroplets on rough hydrophobic surfaces, we find that the Wenzel state is unstable: if the droplet is pressed into complete contact with the substrate (Wenzel-like state) and then let free, it quickly jumps back to the Cassie-like state due to strong thermal fluctuations. For a macroscopic droplet on surfaces with long wavelength roughness, the energetic barrier towards flipping from the Wenzel to the Cassie state may be so large that, even if the Cassie state is the minimum free energy configuration, the system may remains trapped in the (metastable) Wenzel state for all time periods of physical relevance.

\section{Cassie and Wenzel states for randomly rough surfaces}

In this section we discuss under which condition one expects the Cassie state or the Wenzel state to prevail. Consider a rough surface and let $z=h(\mathbf{x})$ be the height of the surface at the point $\mathbf{x}=(x, y)$. A randomly rough surface can be obtained by adding plane waves with random phases:

$$
h(\mathbf{x})=\sum_{\mathbf{q}} B(\mathbf{q}) e^{i[\mathbf{q} \cdot \mathbf{x}+\phi(\mathbf{q})]}
$$

where $\phi(\mathbf{q})$ are independent random variables, uniformly distributed in the interval $[0,2 \pi[$, and with $B(\mathbf{q})=$ $(2 \pi / L)[C(\mathbf{q})]^{1 / 2}$, where $L=A_{0}^{1 / 2}$ is the linear size of 
the surface. The surface roughness power spectrum:

$$
C(\mathbf{q})=\frac{1}{(2 \pi)^{2}} \int d^{2} x\langle h(\mathbf{x}) h(\mathbf{0})\rangle e^{i \mathbf{q} \cdot \mathbf{x}} .
$$

Here $h(\mathbf{x})$ is the surface height profile and $\langle\cdots\rangle$ stands for ensemble average. We have assumed that $\langle h(\mathbf{x})\rangle=0$. We assume that the statistical properties of the rough surface are isotropic, so that $C(\mathbf{q})$ only depends on the magnitude $q=|\mathbf{q}|$ of the wave vector $\mathbf{q}$.

For randomly rough surfaces the normalized surface area $r=A / A_{0}$ is given by (see Appendix):

$$
r=\int_{0}^{\infty} d x\left(1+x \xi^{2}\right)^{1 / 2} e^{-x}
$$

where

$$
\xi^{2}=\int d^{2} q q^{2} C(q)=2 \pi \int_{0}^{\infty} d q q^{3} C(q)=\left\langle(\nabla h)^{2}\right\rangle
$$

is the square of the average slope.

The fraction of the surface where the surface slope $s<$ $s_{0}$ is given by (see Appendix):

$$
P\left(s_{0}\right)=1-e^{-\left(s_{0} / \xi\right)^{2}} .
$$

Note that as $\xi \rightarrow 0$ (corresponding to a flat surface) $P\left(s_{0}\right) \rightarrow 1$ which is expected because the slope of a flat surface is zero and hence smaller than any finite value $s_{0}$. Assume that a liquid exhibits the contact angle $\theta$ on the perfectly flat substrate. The fraction of the surface where the slope $|\nabla h(\mathbf{x})|<|\tan \theta|$ is given by

$$
P(\tan \theta)=1-e^{-(\tan \theta / \xi)^{2}} .
$$

This function is shown in Fig. 8, If we assume that the liquid surface in the liquid-solid non-contact region is flat and parallel to the average substrate surface plane, then we expect the liquid to only occupy the region where the slope is smaller than $\tan \theta$. Note that more than $90 \%$ of the surface area will have a slope below $|\tan \theta|$ if $|\tan \theta| / \xi>1.5$ and in this case the Wenzel state will tend to prevail, while more than $90 \%$ of the surface will have a slope above $|\tan \theta|$ if $|\tan \theta| / \xi<0.3$ and in this case the Cassie state will tend to prevail. For the system we study below $\xi<2$ (see Fig. 12) and for the hydrophobic system $\theta \approx 103^{\circ}$ we get $|\tan \theta| / \xi>2.2$. Thus, one would expect the Wenzel state to prevail. However, the numerical data (see below) tend to suggest that the system is in a Cassielike state. We attribute this to the strong fluctuations at the liquid-solid contact which occurs at the nanoscale, which are particularly important for nanoscale droplets.

\section{SIMULATION METHOD}

We have used Molecular Dynamics (MD) to study the contact angle and contact angle hysteresis. Here we briefly describe the system we studied and how we generated the rough substrate surfaces.

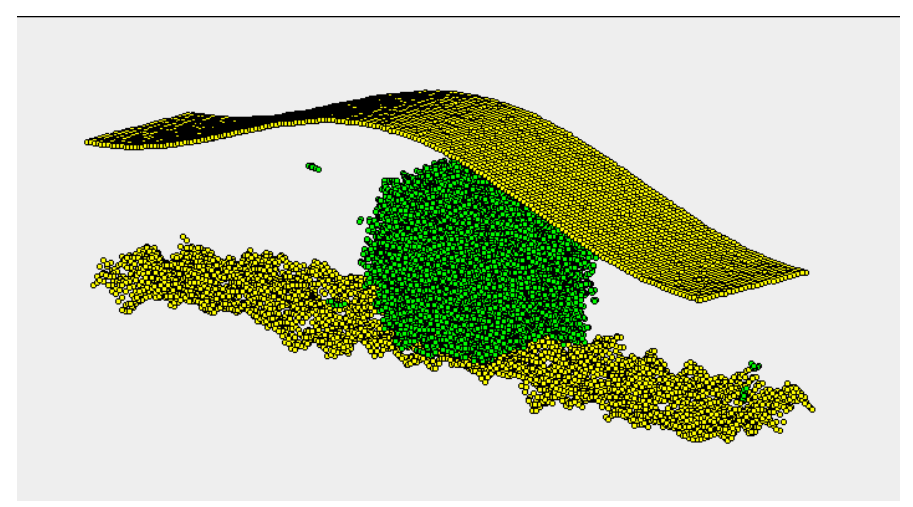

FIG. 9: 3D side-view snapshot of a octane liquid droplet on a hydrophobic and rough substrate. The rigid substrate comprises $200 \times 30$ atoms disposed on a square lattice with lattice constant $a=2.53 \AA$. These atoms have been randomly displaced along the $z$ coordinate, orthogonal to the wall, so to reproduce the desired roughness. The Lennard-Jones solidliquid interaction potential $V(r)=4 \epsilon\left[\left(r_{0} / r\right)^{12}-\left(r_{0} / r\right)^{6}\right]$ with $r_{0}=3.28 \AA, \epsilon=4 \mathrm{meV}$ for hydrophobic substrate and $\epsilon=$ $8 \mathrm{meV}$ for hydrophilic substrate.

\section{A. Molecular dynamics model}

We have used MD calculations to study the influence of surface roughness on liquid droplet contact angle and contact angle hysteresis. We have studied hydrocarbon liquid droplets on different self-affine fractal surfaces. The nanodroplets contained 2364 octane molecules $\mathrm{C}_{8} \mathrm{H}_{18}$ at $T=300 \mathrm{~K}$, which is between the melting and boiling points of octane. The fractal surfaces were generated by adding plane waves with random phases (see Sec. IID and Ref. [41]). Periodic boundary conditions are applied along the $x$ and $y$ directions. The periodically repeated cell forms a rectangle $L_{x} \times L_{y}$ with $L_{x}=506 \AA$ and $L_{y}=75.9 \AA$ (see Fig. 9). The (non-contact) cylindrical droplet diameter is about $104 \AA$, and the size of the droplet-substrate contact area varies (for the hydrophobic system) from $\approx 115 \AA$ (case (a) in Fig. 10) to $\approx 60$ $\AA$ (case (c)).

For most real surfaces usually there is a roll-off wave vector $q_{0}$, below which the power spectrum $C(q)$ of the surface is approximately constant. For $q>q_{0}$ we assume the power spectrum has the power-law behavior $C(q) \sim$ $q^{-2(H+1)}$ [1] corresponding to a self affine fractal surface with the fractal dimension $D_{\mathrm{f}}=3-H$. Different fractal surfaces are obtained by changing the root mean square (rms) roughness amplitude $\sigma$, and the fractal dimension $D_{\mathrm{f}}$. The roll-off wave-vector for the rough surface is $q_{0}=$ $2 \pi / L$ with $L=38 \AA$, and the magnitude of the shortdistance cut-off wave vector $q_{1}=\pi / a$, where $a=2.53$ $\AA$ is the substrate lattice constant. In the present work, we are mainly interested in how the rough structure of the substrate influence wetting. A curved upper wall has been used to speed up the formation of the droplet in the 
initial preparation [42] and to limit the gas-phase volume so that the droplet cannot (fully) evaporate. In some simulations we used a flat upper wall in order to be able to push the droplet towards the substrate a bit more, in order to study the evolution of the receding contact angle.

The lubricant molecules are described through the Optimized Potential for Liquid Simulation (OPLS) [43, 44]; this potential is known to provide density and viscosity of hydrocarbons close to the experimental one. Each octane molecules comprises four units (particles), each particle corresponding to one chemical group $\mathrm{CH}_{2}, \mathrm{CH}_{3}$. The interaction between particles of different molecules is described by Lennard-Jones potentials. The intramolecular interactions include two body forces that keep the bond length $\mathrm{C}-\mathrm{C}$ close to $1.53 \AA$, three body forces imposing a preferred angle of $115^{\circ}$ between the carbon atoms, and four body forces favoring a well defined torsion of the molecules. The four body forces apply to the sequence of carbon atoms $\mathrm{C}-\mathrm{C}-\mathrm{C}-\mathrm{C}[45]$.

We used the Lennard-Jones interaction potential between droplet atoms and substrate atoms. The L.-J. parameters for a hydrophobic surface are chosen such that the Young contact angle is about $100^{\circ}$ when a droplet sits on the flat surface. Because of the periodic boundary conditions and the size of our system, the liquid droplet forms a cylinder with the central line along the $y$-axis, as shown in Figures 9 and 10. We fit the density profile of the droplet to a cylinder (see Fig. 111), and obtain the contact angle $\theta=103^{\circ}$ for the droplet in contact with the flat hydrophobic substrate, while for the flat hydrophilic substrate $\theta=39 \pm 3^{\circ}$.

\section{B. Multiscale rough surfaces}

Many solid surfaces in nature, e.g., surfaces prepared by fracture (involving crack propagation), tend to be nearly self-affine fractal. Self-affine fractal surfaces have multiscale roughness, sometimes extending from the lateral size of the surface down to the atomic scale. A selfaffine fractal surface has the property that if part of the surface is magnified, with a magnification which in general is appropriately different in the directory perpendicular to the surface as compared to the lateral directions, the surface "looks the same" [46] i.e., the statistical properties of the surface are invariant under this scale transformation.

The most important property of a randomly rough surface is the surface roughness power spectrum $C(q)$. We assume that the statistical properties of the surface are translational invariant and isotropic so that $C(q)$ depends only on the magnitude $q=|\mathbf{q}|$ of the wavevector $\mathbf{q}$. For a self-affine surface the power spectrum has the power-law behavior $C(q) \sim q^{-2(H+1)}$, where the Hurst exponent $H$ is related to the fractal dimension $D_{\mathrm{f}}=3-H$. Of course, for real surfaces this relation only holds in some finite wave vector region $q_{0}<q<q_{1}$. Note that in
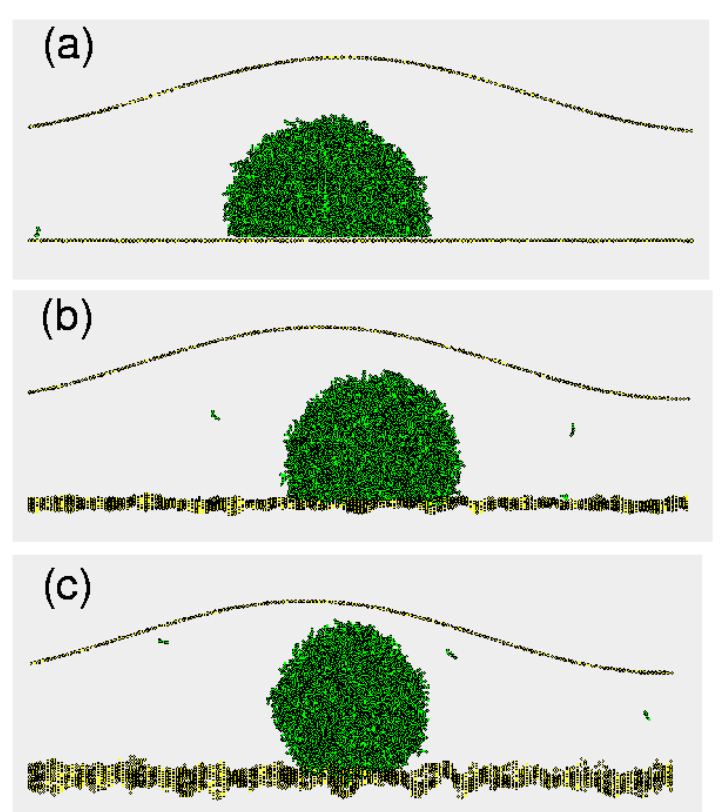

FIG. 10: Snapshots for different root mean square roughness. (a) the droplet is in contact with the flat substrate. (b) and (c) are for rough substrates with the root mean square amplitude $\sigma=2.3 \AA$ and $\sigma=4.8 \AA$, respectively. Adapted from Ref. [1].

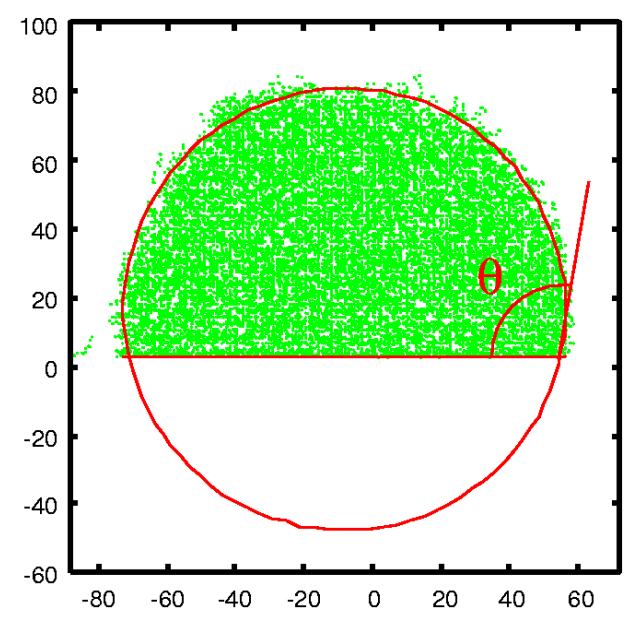

FIG. 11: Determination of the contact angle $\theta$ for the flat substrate. Side view. Adapted from Ref. [11].

many cases there is roll-off wavevector $q_{0}$ below which $C(q)$ is approximately constant. We have generated self affine fractal surfaces by adding plane waves with random phases and appropriately chosen weights, as described in detail in Ref. [41, 46].

In Fig. 12 we show the average slope $\xi$ and the ratio $A / A_{0}$ between the surface area $A$ and the nominal (or projected) $A_{0}$ surface area, as a function of the root mean square roughness $\sigma$ when Hurst exponent $H=0.8$, and 

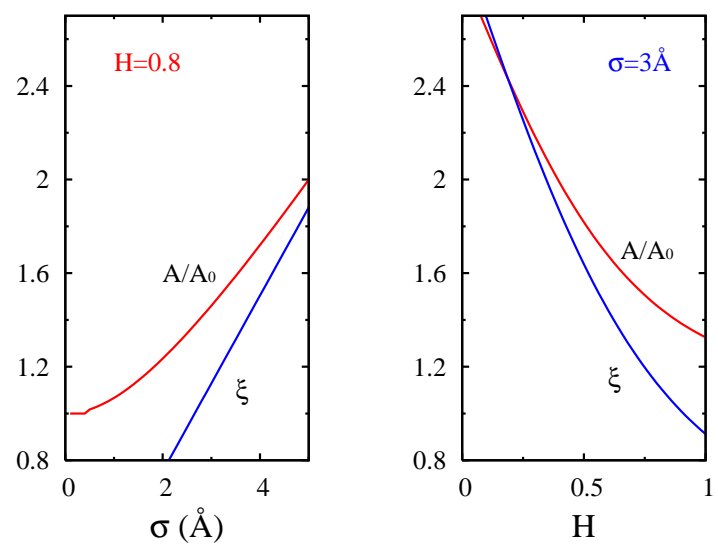

FIG. 12: The average slope $\xi$ and the ratio $A / A_{0}$ between the actual $A$ and the nominal (or projected) $A_{0}$ surface area, as a function of the root mean square roughness $\sigma$ when Hurst exponent $H=0.8$, and as a function of Hurst exponent $H$ for $\sigma=3 \AA$.

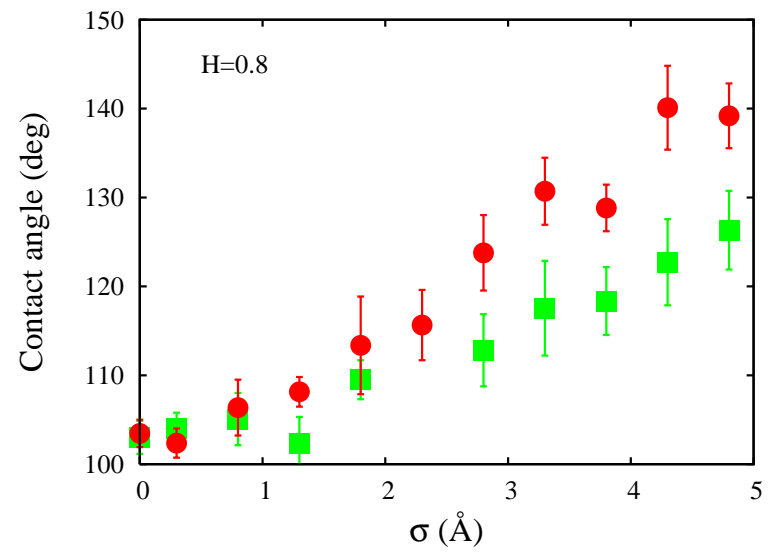

FIG. 13: The contact angle as a function of the root mean square roughness $\sigma$. The circle points are numerical results from the simulations, while the square points are obtained from the Cassie model (see Eq. 7). Each data point is an average over several snap-shot configurations. The fractal dimension is $D_{\mathrm{f}}=2.2$. Adapted from Ref. [11].

as a function of Hurst exponent $H$ for $\sigma=3 \AA$.

\section{NUMERICAL RESULTS}

We present numerical results for the contact angle and contact angle hysteresis for both hydrophilic and hydrophobic systems. The substrate surfaces are assumed to be self affine fractal, but we have varied the fractal dimension and the root-mean-square roughness amplitude.

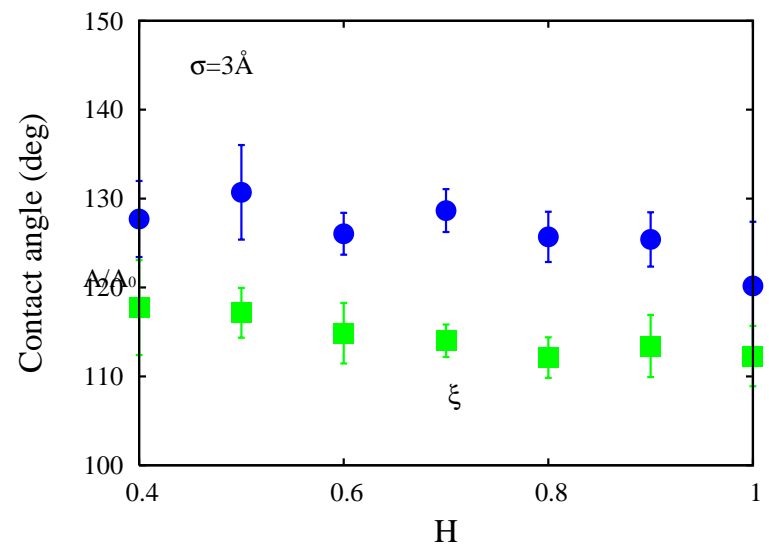

FIG. 14: The contact angle $\theta$ as a function of Hurst exponent $H$ for the rms roughness $\sigma=3 \AA$. The circles and squares have the same meaning as that in Fig. 13 The fractal dimension is $D_{\mathrm{f}}=3-H$. Adapted from Ref. [11].

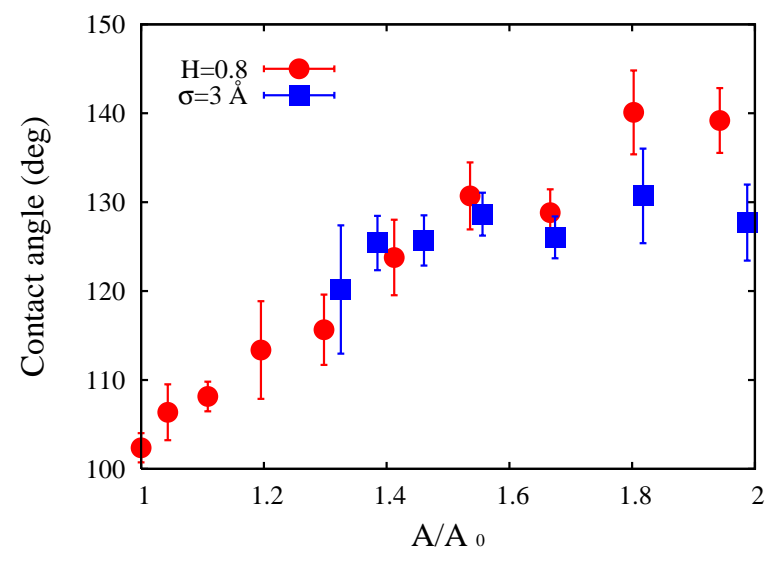

FIG. 15: The contact angle $\theta$ as a function of the normalized surface area $A / A_{0}$ when the root-mean-square amplitude $\sigma$ increases for fixed Hurst exponent $(\mathrm{H}=0.8$, the solid circles) (from Fig. 13) and when the Hurst exponent decreases for a fixed root-mean-square amplitude ( $\sigma=3 \AA$, the solid squares) (from Fig. 14).

\section{A. Static contact angle on hydrophobic surface}

The apparent contact angle, $\theta_{0}$, as a function of the root mean square roughness (rms), is shown in Fig. 13 with the fractal dimension $D_{\mathrm{f}}=2.2$. There is a strong increase in $\theta_{0}$ with increasing rms-roughness amplitude. Fig. 14 shows how $\theta_{0}$ depends on the Hurst exponent $H=3-D_{\mathrm{f}}$. Note that $\theta_{0}$ is almost independent of $H$.

Accordingly to the Wenzel equation, the apparent contact angle $\theta_{0}$ depends only on the surface roughness via the ratio $r=A / A_{0}$. Fig. 12 shows that as $H$ decreases from 1 to 0.4 (i.e., $D_{\mathrm{f}}$ increases from 2 to 2.6 ), $A / A_{0}$ increases by $\sim 50 \%$. However, the MD-calculations show that the apparent contact angle $\theta_{0}$ is almost independent 

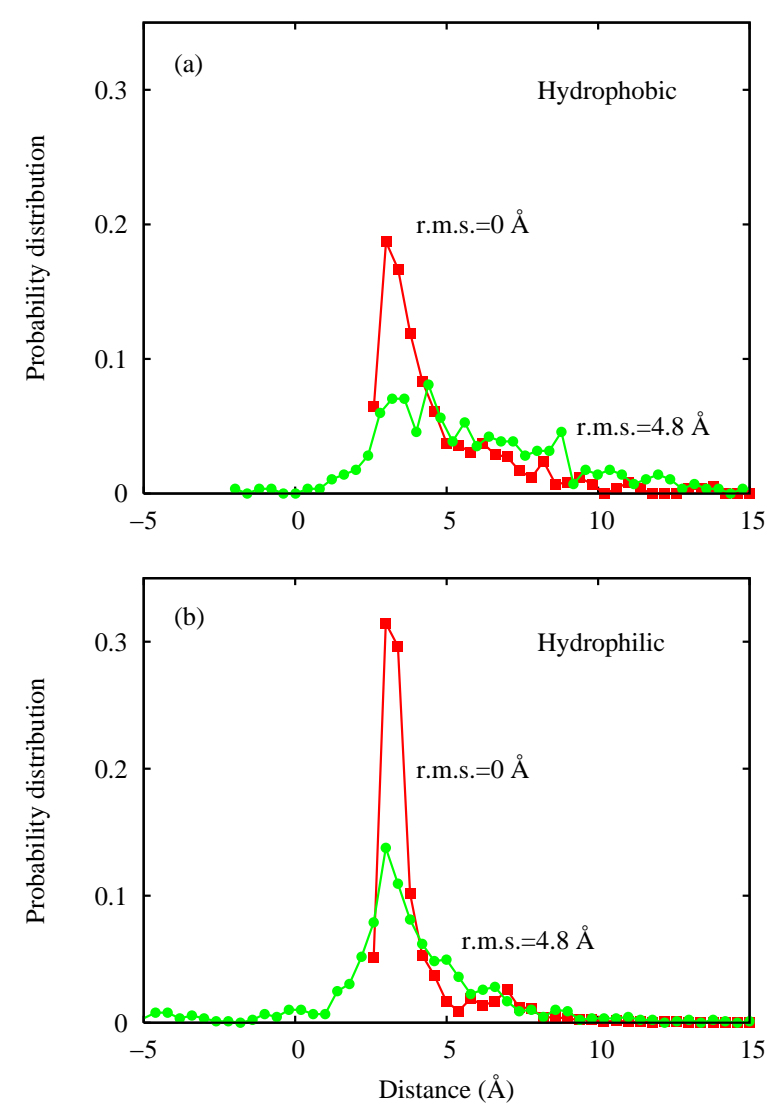

FIG. 16: (a) The height probability distribution for hydrophobic surface both flat (squares) and rough (circles). (b) The height probability distribution for hydrophilic surface both flat (squares) and rough (circles).

of the fractal dimension, see Fig. 14. This is also illustrated in Fig. 15 which shows the contact angle as a function of the (normalized) surface area $A / A_{0}$ for both cases. Thus the Wenzel equation cannot be used in the present situation. This is consistent with a visual inspection of the liquid-substrate interface which shows that on the rough substrates, the droplet is "riding" on the asperity tops of the substrate, i.e., the droplet is in a Cassie-like state. In order to quantitatively verify this, we have calculated the distances $u(x, y)$ between the bottom surface of the liquid drop and the rough substrate surface in the (apparent) contact area. From the distribution[47]

$$
P(u)=\langle\delta[u-u(x, y)]\rangle
$$

of these distances [see Fig. 16(a)] we obtain the fraction $\psi$ of the (projected) surface area where contact occurs:

$$
\psi=\int_{0}^{u_{1}} d u P(u)
$$

where $u_{1}$ is a cut-off distance to distinguish between contact and no-contact regions, which has to be comparable to the typical bond distance (we use $u_{1}=4 \AA$ ). Note that

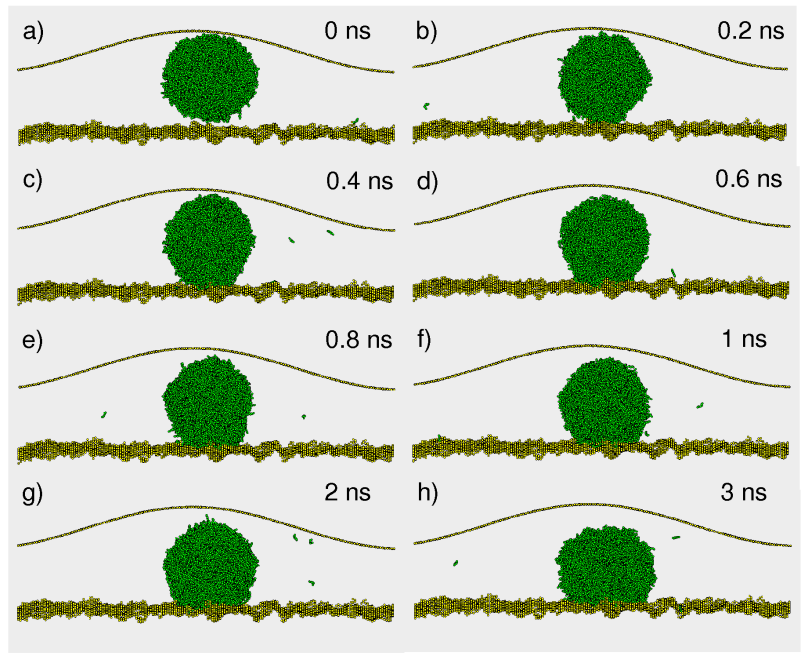

FIG. 17: The advancing contact angle $\theta_{\mathrm{a}}$ evolution for hydrophobic nanodroplet. $\theta_{\mathrm{a}}$ is measured when the solid/liquid contact area increases.

due to the thermal fluctuations $\psi=\psi_{0}$ for flat surface is less than 1. Using the normalized $\phi=\psi / \psi_{0}$, the Cassie model (with $r=1$ ) predicts the variation of the contact angle with $\sigma$ and $H$ given in Fig. 13 and 14 (square points).

Fig. 13 shows that the apparent contact angle $\theta_{0}$ increases strongly with increasing rms-roughness amplitude, at fixed fractal dimension $D_{\mathrm{f}}=2.2$, while it is nearly independent of the fractal dimension $D_{\mathrm{f}}$ (see Fig. 14). Since increasing the fractal dimension at constant rms-roughness amplitude mainly increases the short-wavelength roughness, we conclude that the nanoscale wavelength roughness doesn't matter so much in determining the contact angle for hydrophobic surfaces, while the long wavelength roughness plays an important role. We attribute this fact to the strong thermal fluctuations in the height (or width) $u$ of the liquidsolid interface which occur at the nanoscale even for the flat substrate surface. Note also that in our model the wall-wall interaction is long-ranged, decaying effectively as $\sim 1 / u^{3}$, so there will be a contribution to the interfacial energy also for non-contacting surfaces which, of course, is not rigorously included in the macroscopic Cassie model.

\section{B. Dynamic contact angle: Contact angle hysteresis}

The advancing contact angle $\theta_{\mathrm{a}}$ is measured when the solid/liquid contact area increases, while the receding contact angle $\theta_{\mathrm{r}}$ is measured when the contact area shrinks. If the difference $\theta_{\mathrm{a}}-\theta_{\mathrm{r}}$ is nonzero, the liquidsubstrate system exhibits contact angle hysteresis. 


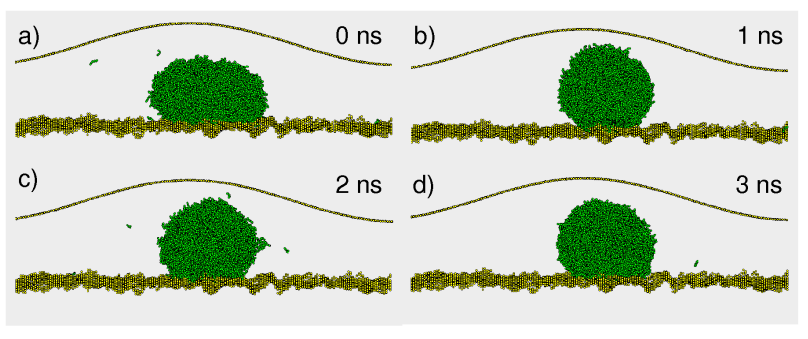

FIG. 18: The receding contact angle $\theta_{\mathrm{r}}$ evolution for hydrophobic nanodroplet. $\theta_{\mathrm{r}}$ is measured when the solid/liquid contact area shrinks.

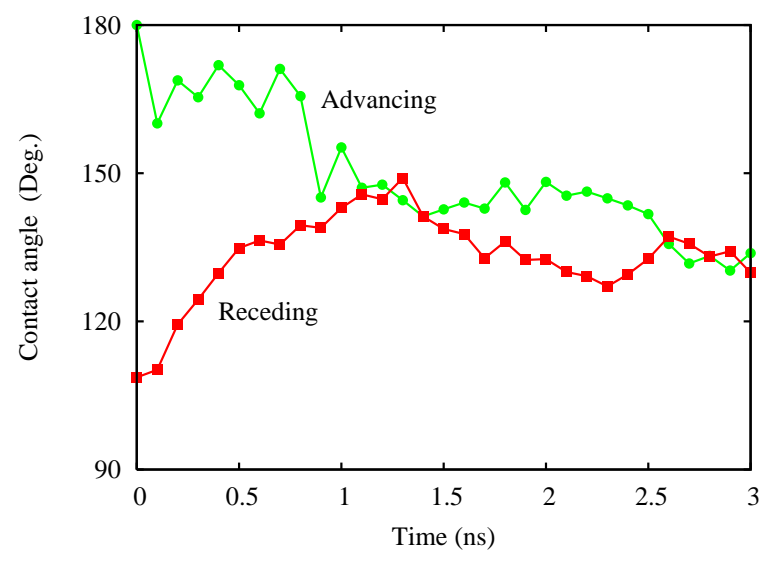

FIG. 19: The advancing (circles) and receding (squares) contact angle $\theta$, for hydrophobic substrate, as a function of time. The root-mean-square roughness of the substrate is $\mathrm{rms}=4.8$ $\AA$ A. $\epsilon=4 \mathrm{meV}$ and $r_{0}=3.28 \AA$. The thermal equilibrium contact angle has been reached after a few nanoseconds, irrespective of whether the initial contact angle is larger or smaller than the equilibrium angle.

\section{Hydrophobic surfaces}

Figures 17 and 18 show the time-evolution of the advancing contact angle $\theta_{\mathrm{a}}$ and receding contact angle $\theta_{\mathrm{r}}$, respectively, for a nanodroplet on a rough, hydrophobic substrate. The former has been obtained by placing the droplet close to the substrate, so that the drop will spontaneously spread under the adhesive interaction with the substrate. The contact angle evolves in time from $\theta=180^{\circ}$ (non-contact) in panel 17(a) to its asymptotic value $\theta_{a}=140^{\circ}$, reached after $3 \mathrm{~ns}$ in panel $17(\mathrm{~h})$.

The receding contact angle was simulated by squeezing the droplet into a pancake-like shape with the upper wall. The interaction between the atoms of the upper wall and drop atoms is given by the repulsive term of a LennardJones potential, i.e. $V(r)=4 \epsilon_{0}\left(r_{0} / r\right)^{12}$. The lack of attraction with the top surface allows us to suddenly pull the wall up, leaving the drop in the configuration of panel 18(a). The free drop increases the contact angle up to the asymptotic value $\theta_{r}$. Figure 19 shows the time evo-
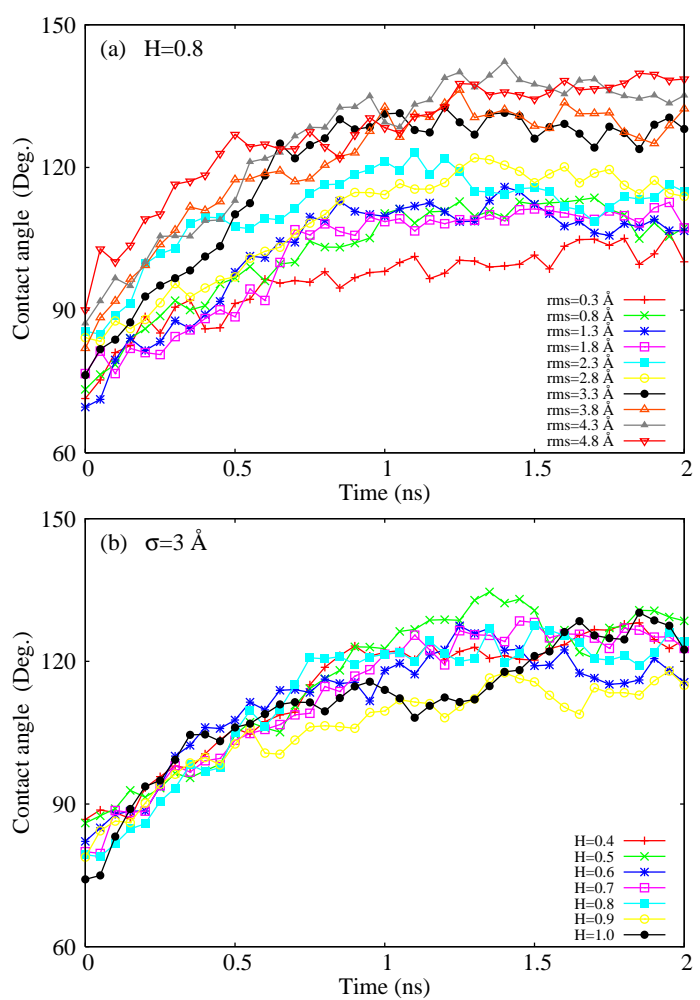

FIG. 20: The time evolution of the receding contact angle for the hydrophobic droplets, on the substrates with (a) various root-mean-square (rms) roughness while the Hurst exponent $\mathrm{H}=0.8$, and (b) various Hurst exponent $\mathrm{H}$ while the rootmean-square roughness $(\mathrm{rms}=3 \AA$ ).

lution of the contact angle for these two cases. Note the strong time oscillations of the contact angle which are due to the rearrangement of the liquid molecules at the solid-liquid interface. The corresponding energy barriers are small compared to the thermal fluctuations (see Sec. IIC). However, after a few nanoseconds we find that both the receding and advancing contact angle fluctuate around the same average value; thus no contact angle hysteresis is observed for the hydrophobic system.

To be sure that in any system there is no contact angle hysteresis, we performed extensive simulations on various substrates with different root-mean-square roughness amplitudes (rms) (see Fig. 20(a)). and with different Hurst exponents $H$ (see Fig.20(b)), The receding contact angle reaches its asymptotic value within about 2 or 3 nanoseconds. In Figure 20 one can see a relatively broad range of receding contact angles for substrates wih different rms roughness. Conversely, substrates with different Hurst exponents show nearly the same contact angle. The root-mean-square roughness is mainly determined by long wavelength roughness of the surface. Increasing the fractal dimension $D_{\mathrm{f}}$ signifies that the short wavelength roughness increases. Thus, one can see that the contact angle is more sensitive to the long wavelength roughness of the substrate than to the short wavelength roughness. 


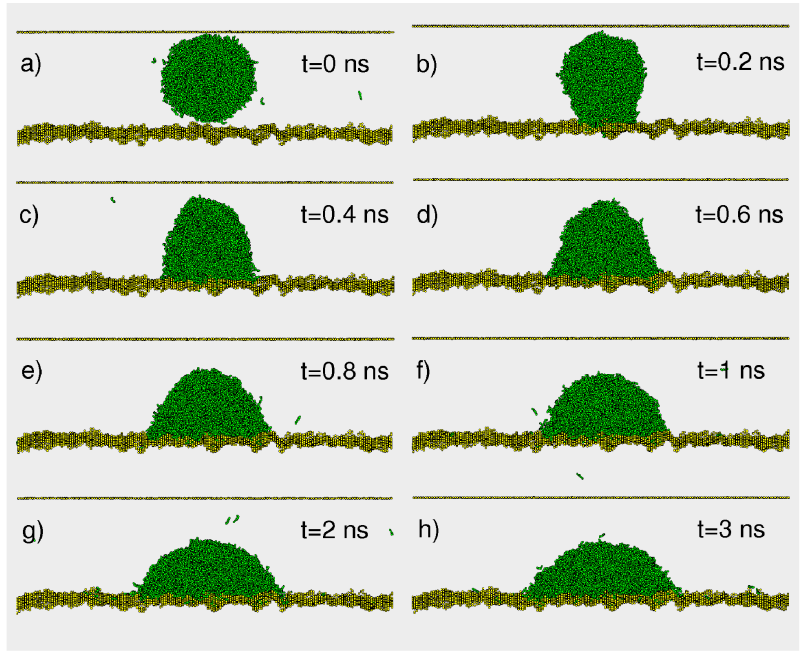

FIG. 21: Advancing contact angle evolution for hydrophilic nanodroplet. The root-mean-square roughness of the substrate is rms $=4.8 \AA$. The energy parameter and the equilibrium distance in L.-J. potential are $\epsilon=8 \mathrm{meV}$ and $r_{0}=3.28$ $\AA$.

This agrees with the results in Fig. 13 and 14

A comparison of these results with those of the corresponding simulations for the advancing contact angle, confirms that there is no hysteresis. This is in drastic contrast to simulation studies we have performed for hydrophilic surfaces (see below), where surface roughness results in strong pinning of the boundary line; for such surfaces it is therefore impossible to study static droplet contact angles (as observed on macroscopic time scales) using molecular dynamics.

Comparing the form of $P(u)$ for the flat and the most rough surfaces shows that the system is in a Cassie-like state, but at the nanoscale the difference between the Cassie state and the Wenzel state is not so large due to the thermal fluctuations. That is, already for the flat surface strong thermal fluctuations at the interface result in nanosized regions where the separation between the solid and the liquid is much larger than the natural (lowtemperature) binding separation. When the substrate is rough the fluctuations become even larger and the system is in a state which is more Cassie-like than Wenzel-like. This also explains why no hysteresis is observed: The Wenzel state is probably the (low temperature) energy minimum state (see Sec. IID), but squeezing the droplet into a pancake shape does not push the system permanently into the Wenzel state (where pinning effects may be very important) because even if it would go into this state temporarily, the free energy barrier separating the Cassie and Wenzel states is so small that thermal fluctuations would quickly kick it back to the Cassie-like state.

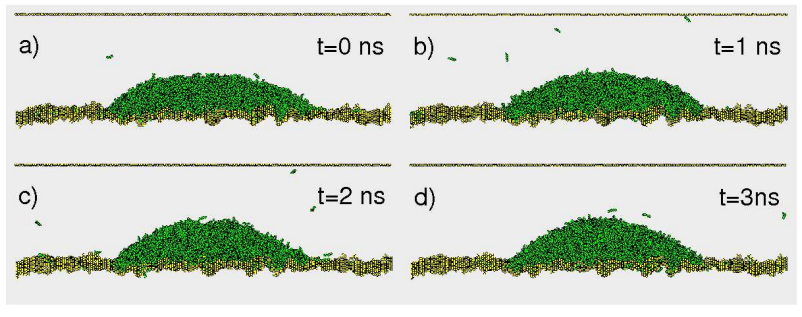

FIG. 22: Receding contact angle evolution for hydrophilic nanodroplet. The root-mean-square roughness of the substrate is rms $=4.8 \AA$. The energy parameter and equilibrium parameter in L.-J. potential are $\epsilon=8 \mathrm{meV}$ and $r_{0}=3.28 \AA$.

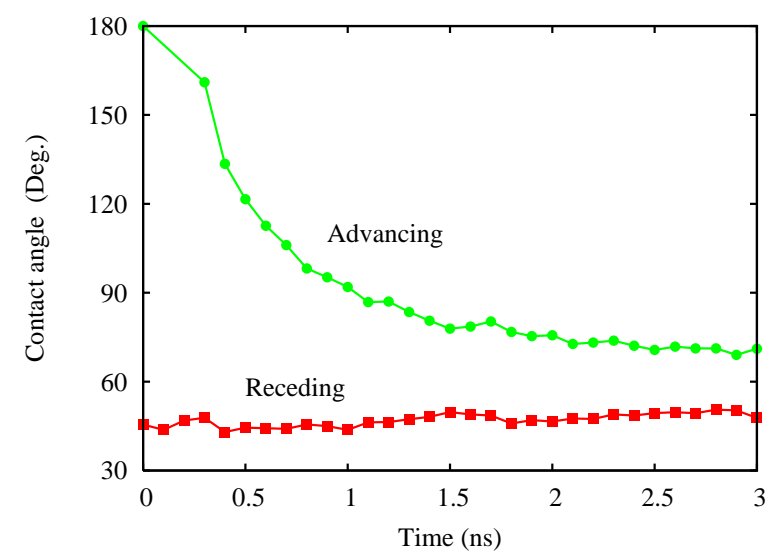

FIG. 23: The advancing (circles) and receding (squares) contact angle $\theta$, for the hydrophilic substrate, as a function of time. The root-mean-square roughness of the substrate is $\mathrm{rms}=3 \AA$, and the L.-J. substrate-liquid interaction parameters $\epsilon=8 \mathrm{meV}$ and $r_{0}=3.28 \AA$.

\section{Hydrophilic surfaces}

Let us now consider the case where the liquid droplet contact angle on the flat surface $\theta<90^{\circ}$ (hydrophilic system). We choose the energy parameter and the equilibrium distance in L.-J. potential, associated with the liquid-solid atom interaction, as $\epsilon=8 \mathrm{meV}, r_{0}=3.28 \AA$ respectively. This gives $\theta \approx 70^{\circ}$. Figures 21,22 and 23 for hydrophilic droplet, are analogous to Figures [17. 18 and 19 respectively, for hydrophobic droplet. In Fig. 21 we show the time dependence of the advancing contact angle for the hydrophilic nanodroplet. The root-meansquare roughness of the substrate is $\mathrm{rms}=4.8 \AA$.

In Fig. 22 we show the time evolution of the receding contact angle for the hydrophilic nanodroplet.

Fig. 23 shows the advancing (circles) and receding (squares) contact angle $\theta$ as a function of time, on a hydrophilic substrate with root-mean-square roughness rms $=3 \AA$. Note that the thermal equilibrium contact angle cannot be reached on the time-scale of the simulations. Note also that the fluctuation in the contact 


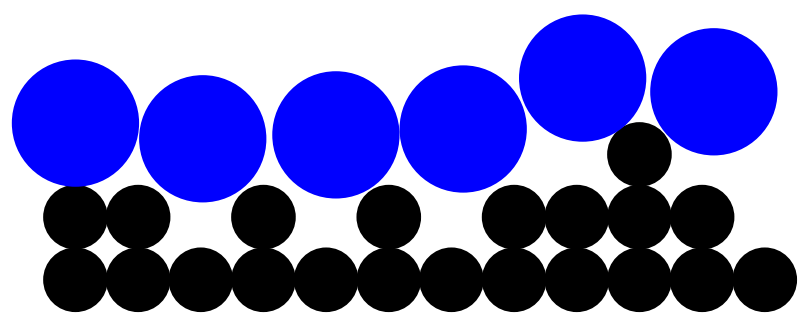

FIG. 24: The natural separation between the molecules in a liquid is usually considerably larger than the atom-atom separation on the substrate surface. This implies that the fluid molecules cannot "follow" the atomic scale roughness so that the fluid molecules will naturally be in a Cassie-like state with respect to the shortest substrate roughness components determined by the substrate nearest neighbor atom-atom separation.

angle are much smaller than for the hydrophobic system (Fig. 19). This is, of course, due to the fact that for the hydrophilic system the liquid-substrate interaction is much stronger, and the barriers for the rearrangement of liquid molecules at the substrate-liquid interface much higher than for the hydrophobic system.

Finally, in Fig. 16(b) we show the height probability distribution for the hydrophilic surface for both the flat (squares) and rough (circles) hydrophilic surface. Note that the fluctuations in the liquid-solid separation at the interface is much smaller on the hydrophilic surface than on the hydrophobic surface [16(a)].

\section{DISCUSSION}

In most practical cases it is not possible to modify the surface roughness without simultaneously affecting the chemical nature of the surface. While this is obvious for crystalline materials, where surface roughening will result in the exposure of new lattice planes with different intrinsic surface energy, it may also hold for amorphouslike materials, where surface roughening may result in a more open atomic surface structure, with an increased fraction of (weak) unsaturated bonds. In our model study a similar effect occurs, and some fraction of the change in the contact angle with increasing root-mean-square amplitude may be associated with this effect. However, the most important result of our study, namely that the contact angle is mainly determined by the long-wavelength roughness, should not be affected by this fact.

Another reason for why the short-wavelength (atomic) roughness may influence the liquid contact state differently from long-wavelength roughness has to do with the fact that the natural separation between the molecules in a liquid is usually considerably larger than the atomatom separation on the substrate surface. This implies that the fluid molecules cannot "follow" the atomic scale roughness (see Fig. 24) so that the fluid molecules will naturally be in a Cassie-like state with respect to the shortest substrate roughness wavelength components, determined by the substrate nearest neighbor atom-atom separation.

It is important to note that the discussion in this paper is also relevant for the contact between macroscopic liquid drops and rough substrates. That is, if the solidliquid interface is studied with nanometer resolution $\lambda$, then the strong fluctuations (in time and space) at the liquid-solid interface discussed above will also be observed for a macroscopic droplet, and the interfacial energy $\gamma_{\mathrm{sl}}(\zeta)$ for $\zeta=D / \lambda$ (where $D$ is the diameter of the nominal contact area between the (macroscopic) liquid droplet and the substrate) will be the same as obtained above for nanodroplets at the same resolution $\lambda$.

\section{SUMMARY AND CONCLUSION}

We have discussed under which condition the Wenzel or Cassie state is favorable on randomly rough surfaces. We performed molecular dynamics simulations to study contact angle and the contact angle hysteresis on hydrophobic and hydrophilic surfaces. The contact angle on hydrophobic surfaces depends strongly on the rootmean-square roughness of the substrate, but is nearly independent of the fractal dimension. For hydrophobic surfaces, there is no contact angle hysteresis due to strong thermal fluctuations at the nanoscale. For hydrophilic surfaces we observe contact angle hysteresis due to pinning effects resulting from the much higher energy barriers for rearrangement of liquid molecules at the solidliquid interface. This indicates that on randomly rough hydrophobic surfaces the Cassie-like state often prevails, at least for nanoscale droplets. We have found that thermal fluctuations play an important role at the nanoscale, which leads to the enhanced hydrophobicity by surface roughness. It is of particular importance to design and build superhydrophobic surfaces.

\section{APPENDIX: DISTRIBUTION OF SURFACE SLOPES FOR RANDOMLY ROUGH SURFACES}

In this appendix we derive the distribution of surface slopes for randomly rough surfaces. We discuss under which conditions one expects the Wenzel and Cassie states to prevail.

The surface area $A$ and the average surface slope $\xi$

Consider a randomly rough surface and let $h(\mathbf{x})$ denote the height profile measured from the average plane so that $\langle h(\mathbf{x})\rangle=0$, where $\langle.$.$\rangle stands for ensemble averag-$ ing, or (equivalently) averaging over the surface area. We assume that $h(\mathbf{x})$ is a Gaussian random variable charac- 
terized by the power spectrum

$$
C(q)=\frac{1}{(2 \pi)^{2}} \int d^{2} x\langle h(\mathbf{x}) h(\mathbf{0})\rangle e^{-i \mathbf{q} \cdot \mathbf{x}} .
$$

Note that if we write

$$
h(\mathbf{x})=\int d^{2} q h(\mathbf{q}) e^{i \mathbf{q} \cdot \mathbf{x}}
$$

where

$$
h(\mathbf{q})=\frac{1}{(2 \pi)^{2}} \int d^{2} x h(\mathbf{x}) e^{-i \mathbf{q} \cdot \mathbf{x}}
$$

then

$$
\left\langle h(\mathbf{q}) h\left(\mathbf{q}^{\prime}\right)\right\rangle=\delta\left(\mathbf{q}+\mathbf{q}^{\prime}\right) C(q) .
$$

Sometimes it is also convenient to use

$$
\langle h(\mathbf{q}) h(-\mathbf{q})\rangle=\frac{A_{0}}{(2 \pi)^{2}} C(q),
$$

where $A_{0}$ is the surface area. In deriving (A2) we have used that

$$
\delta(\mathbf{q}-\mathbf{q})=\frac{1}{(2 \pi)^{2}} \int d^{2} x e^{i(\mathbf{q}-\mathbf{q}) \cdot \mathbf{x}}=\frac{A_{0}}{(2 \pi)^{2}} .
$$

If the surface roughness amplitudes $h(\mathbf{q})$ are assumed to be Gaussian random variables, one can show that the (normalized) surface area 48]

$$
r=\frac{A}{A_{0}}=\int_{0}^{\infty} d x\left(1+x \xi^{2}\right)^{1 / 2} e^{-x}
$$

where

$$
\xi^{2}=\int d^{2} q q^{2} C(q)=2 \pi \int_{0}^{\infty} d q q^{3} C(q) .
$$

Let us calculate the rms surface slope. We get

$$
\left\langle(\nabla h)^{2}\right\rangle=\int d^{2} q d^{2} q^{\prime}\left(i q_{\alpha}\right)\left(i q_{\alpha}^{\prime}\right)\left\langle h(\mathbf{q}) h\left(\mathbf{q}^{\prime}\right)\right\rangle e^{i\left(\mathbf{q}+\mathbf{q}^{\prime}\right) \cdot \mathbf{x}}
$$

Using (A1) this gives

$$
\left\langle(\nabla h)^{2}\right\rangle=\int d^{2} q q^{2} C(q)=\xi^{2}
$$

Thus, for a Gaussian random surface both the average slope and the increase in the surface area is determined by the parameter $\xi$. For non-random surfaces this is no longer the case.

\section{Surface slope probability distribution}

Let $h(\mathbf{x}, \zeta)$ denote the height profile after having smoothed out surface roughness with wavelength shorter than $\lambda=L / \zeta$. For example, we may define

$$
h(\mathbf{x}, \zeta)=\int_{q<q_{1}} d^{2} q h(\mathbf{q}) e^{i \mathbf{q} \cdot \mathbf{x}},
$$

where $q_{1}=q_{L} \zeta\left(\right.$ where $\left.q_{L}=2 \pi / L\right)$. We will refer to $\zeta$ as the magnification. Thus, when we study the surface at the magnification $\zeta$ we will only detect surface roughness with wavelength components larger than $\lambda=L / \zeta$.

We will now derive an equation of motion for the surface slope probability distribution function

$$
P(\mathbf{s}, \zeta)=\langle\delta(\mathbf{s}-\nabla h(\mathbf{x}, \zeta))\rangle .
$$

We assume that the surface roughness amplitudes $h(\mathbf{q})$ are independent random variables. In this case, if we write

$$
h(\mathbf{x}, \zeta+\delta \zeta)=h(\mathbf{x}, \zeta)+\delta h,
$$

we get

$$
P(\mathbf{s}, \zeta+\delta \zeta)=\langle\delta(\mathbf{s}-\nabla h(\mathbf{x}, \zeta+\delta \zeta))\rangle
$$$$
=\int d^{2} s^{\prime}\left\langle\delta\left(\mathbf{s}^{\prime}-\nabla \delta h\right)\right\rangle\left\langle\delta\left(\mathbf{s}-\mathbf{s}^{\prime}-\nabla h(\mathbf{x}, \zeta)\right)\right\rangle
$$

$$
=\int d^{2} s^{\prime}\left\langle\delta\left(\mathbf{s}^{\prime}-\nabla \delta h\right)\right\rangle P\left(\mathbf{s}-\mathbf{s}^{\prime}, \zeta\right)
$$

But

$$
\begin{aligned}
& \left\langle\delta\left(\mathbf{s}^{\prime}-\nabla \delta h\right)\right\rangle=\frac{1}{(2 \pi)^{2}} \int d^{2} q\left\langle e^{i \mathbf{q} \cdot\left(\mathbf{s}^{\prime}-\nabla \delta h\right)}\right\rangle \\
& =\frac{1}{(2 \pi)^{2}} \int d^{2} q\left(1-\frac{1}{2}\left\langle(\mathbf{q} \cdot \nabla \delta h)^{2}\right\rangle\right) e^{i \mathbf{q} \cdot \mathbf{s}^{\prime}}
\end{aligned}
$$

$$
=\delta\left(\mathbf{s}^{\prime}\right)+\frac{1}{2}\left\langle\left(\nabla_{\alpha} \delta h\right)\left(\nabla_{\beta} \delta h\right)\right\rangle \frac{\partial}{\partial s_{\alpha}^{\prime}} \frac{\partial}{\partial s_{\beta}^{\prime}} \delta\left(\mathbf{s}^{\prime}\right) .
$$

Substituting this result into (A3) and expanding the lefthand-side to linear order in $\delta \zeta$ gives

$$
\frac{\partial P}{\partial \zeta}(\mathbf{s}, \zeta)=\frac{1}{2 \delta \zeta}\left\langle\left(\nabla_{\alpha} \delta h\right)\left(\nabla_{\beta} \delta h\right)\right\rangle \frac{\partial}{\partial s_{\alpha}} \frac{\partial}{\partial s_{\beta}} P(\mathbf{s}, \zeta)
$$

But

$$
\begin{gathered}
\left\langle\left(\nabla_{\alpha} \delta h\right)\left(\nabla_{\beta} \delta h\right)\right\rangle=\int_{q_{L} \zeta}^{q_{L}(\zeta+\delta \zeta)} d^{2} q d^{2} q^{\prime} \\
\times\left(i q_{\alpha}\right)\left(i q_{\beta}^{\prime}\right)\left\langle h(\mathbf{q}) h\left(\mathbf{q}^{\prime}\right)\right\rangle e^{\left(\mathbf{q}+\mathbf{q}^{\prime}\right) \cdot \mathbf{x}} \\
=\int_{q_{L} \zeta}^{q_{L}(\zeta+\delta \zeta)} d^{2} q q_{\alpha} q_{\beta} C(q) \\
=\frac{1}{2} \delta_{\alpha \beta} \int_{q_{L} \zeta}^{q_{L}(\zeta+\delta \zeta)} d^{2} q q^{2} C(q)
\end{gathered}
$$




$$
=\pi \delta_{\alpha \beta} q_{L} \delta \zeta q^{3} C(q)
$$

Thus

$$
\frac{1}{2 \delta \zeta}\left\langle\left(\nabla_{\alpha} \delta h\right)\left(\nabla_{\beta} \delta h\right)\right\rangle=\frac{\pi}{2} \delta_{\alpha \beta} q_{L} q^{3} C(q)
$$

Substituting this result in (A4) gives the following diffusion-like equation for $P(\mathbf{s}, \zeta)$ :

$$
\frac{\partial P}{\partial \zeta}=D(\zeta) \nabla_{s}^{2} P
$$

where

$$
\nabla_{s}^{2}=\frac{\partial}{\partial s_{\alpha}} \frac{\partial}{\partial s_{\alpha}}
$$

where the "diffusivity"

$$
D(\zeta)=\frac{\pi}{2} q_{L} q^{3} C(q),
$$

with $q=q_{L} \zeta$.

\section{Solution of the diffusion equation}

The function $P(\mathbf{s}, \zeta)$ describes the probability to observe a surface slope or gradient $\mathbf{s}=\nabla h(\mathbf{x})$ when the system is studied at the magnification $\zeta$. When the system is studied at the lowest magnification $\zeta=1$ the surface appears flat and smooth so that the gradient vanishes, i.e.,

$$
P(\mathbf{s}, 1)=\langle\delta(\mathbf{s}-\nabla h(\mathbf{x}, 1))\rangle=\delta(\mathbf{s}) .
$$

We also require that there is no infinite high slopes, i.e.,

$$
P(\mathbf{s}, \zeta) \rightarrow 0 \text { as }|\mathbf{s}| \rightarrow \infty .
$$

Let us determine the solution to (A5) which obeys the "initial" condition (A7) and the boundary condition (A8). It is clear that the solution is given by

$$
P(\mathbf{s}, \zeta)=\frac{1}{\pi s_{1}^{2}} e^{-\left(s / s_{1}\right)^{2}},
$$

where the width parameter $s_{1}(\zeta)$ depends on the magnification:

$$
s_{1}^{2}=4 \int_{1}^{\zeta} d \zeta^{\prime} D\left(\zeta^{\prime}\right)=2 \pi \int_{q_{L}}^{\zeta q_{L}} d q q^{3} C(q)=\xi^{2}(\zeta) .
$$

Note that $P$ is normalized,

$$
\int d^{2} s P(\mathbf{s}, \zeta)=1
$$

and that the width of the Gaussian distribution $P$ increases with increasing resolution, i.e., when the surface is studied at higher and higher resolution, steeper and steeper surface slopes will be detected.

Distribution function $P\left(s_{0}, \zeta\right)$

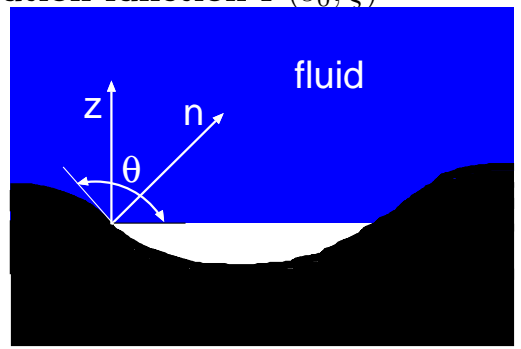

FIG. 25: A fluid in contact with a rough substrate. The contact angle $\theta$ is determined by Young's equation.
In what follows we will need the fraction $P\left(s_{0}, \zeta\right)$ of the total surface area where the slope $s<s_{0}$, where $s_{0}$ is a fixed number between zero and infinite. Let $A_{\perp}(\zeta)$ be the surface area, projected on the $x y$-plane, where the surface slope $s<s_{0}$. We then have

$$
P\left(s_{0}, \zeta\right)=A_{\perp}(\zeta) / A_{0}
$$

Using the definition

$$
P(\mathbf{s}, \zeta)=\langle\delta(\mathbf{s}-\nabla h(\mathbf{x}, \zeta))\rangle
$$

$$
=\frac{1}{A_{0}} \int d^{2} x \delta(\mathbf{s}-\nabla h(\mathbf{x}, \zeta))
$$

we get

$$
P\left(s_{0}, \zeta\right)=\int_{s<s_{0}} d^{2} s P(\mathbf{s}, \zeta)
$$

Using (A9) this gives

$$
P\left(s_{0}, \zeta\right)=1-e^{-\left(s_{0} / s_{1}\right)^{2}} .
$$

\section{Surface area with slope below $\tan \theta$}

Consider a liquid in contact with a rough substrate. The contact angle $\theta$ is determined by Young's equation:

$$
\cos \theta=\frac{\gamma_{\mathrm{sv}}-\gamma_{\mathrm{sl}}}{\gamma_{\mathrm{lv}}}
$$

where $\gamma_{\mathrm{sl}}, \gamma_{\mathrm{sv}}$ and $\gamma_{\mathrm{lv}}$ are the solid-liquid, solid-vapor and liquid-vapor interfacial energies, respectively. Note that if $\mathbf{n}$ is the normal to the solid surface and $\mathbf{z}$ the normal to the liquid surface, which we assume to be parallel to the average surface plane (see Fig. 25), then $\cos \theta=-\mathbf{z} \cdot \mathbf{n}$. Since

$$
\mathbf{n}=\frac{(-\nabla h, 1)}{\left(1+(\nabla h)^{2}\right)^{1 / 2}}
$$

we get

$$
\cos \theta=-\left(1+(\nabla h)^{2}\right)^{-1 / 2}, \quad|\tan \theta|=|\nabla h| .
$$

Thus, using (A10) the fraction of the surface where the surface slope is below $\tan \theta$ is

$$
P(\tan \theta)=1-e^{-(\tan \theta / \xi)^{2}}
$$


[1] T. Young, Phil. Trans. R. Soc. Lond. 95 (1805), 65

[2] P.S. Laplace, OEUVRES, (Paris: Imprimerie Royale), (1847)

[3] J. Rowlinson, B. Widom, Molecular Theory of Capillarity, (Oxford: Oxford University Press), (1982)

[4] P.G. de Gennes, Rev. Mod. Phys. 57, No.3, Part I, 827 (1985)

[5] D. Quere, Rep. Prog. Phys. 68, 2495 (2005)

[6] T.S. Chow, J. Phys.: Condens. Matter 10, L445 (1998)

[7] N.A. Patankar, Langmuir, 19, 1249 (2003)

[8] W. Chen, et. al, Langmuir 15, 3395 (1999).

[9] M. Callies and D. Quere, Soft Matter, 1, 55 (2005)

[10] L.W. Schwartz and S. Garoff, Langmuir, 1, 219 (1985)

[11] C. Yang, U. Tartaglino, B.N.J. Persson, Phys. Rev. Lett. 97 116103, (2006)

[12] C.W. Extrand,S.I. Moon, P. Hall and D. Schmidt, Langmuir, 23, 8882 (2007)

[13] C. Yang, U. Tartaglino and B.N.J. Persson, J. Phys.: Condens. Matter 50, 11521 (2006)

[14] S.L. Ren, S.R. Yang, Y.P. Zhao, T.X. Yu and X.D. Xiao, Surf. Sci. 546, 64 (2003)

[15] M. Nosonovsy, B. Bhushan, Microsystem TechnologiesMicro- and Nanosystem-Information storage and Processing Systems, 11: 535 (2005)

[16] See http://www.lotus-effect.com for information involving surface roughness in relation to hydrophobicity and surface self-cleaning in biological systems.

[17] W. Barthlott, C. Neinhuis, Planta 202, 1 (1997)

[18] C. Neinhuis, W. Barthlott, Annals of Botany 79, 667 (1997)

[19] A. Otten, S. Herminghaus, Langmuir 20, 2405 (2004)

[20] S. Schibuichi, T. Onda, N. Satoh, K. Tsujii, J. Phys. Chem. 100, 19512 (1996)

[21] R. Blossey, Nature Mat. 2 301, (2003)

[22] N.A. Patankar, Langmuir 20, 8209 (2004)

[23] Y.T. Cheng, D.E. Rodak, Appl. Phys. Lett. 86144101 (2005)

[24] A. Nakajima, K. Hashimoto, T. Watanabe, Monatsh. Chem. 132, 31 (2001)

[25] S.R. Coulson, L. Woodward, J.P.S. Badyal, S.A. Brewer, C. Willis, J. Phys. Chem. B 104, 8836 (2000)

[26] H.Y. Erbil, A.L. Demirel, Y. Avci, O. Mert, Science 299, 1377 (2003)

[27] R.N. Wenzel, Ind. Eng. Chem. 28, 988 (1936)

[28] A.B.D. Cassie, S. Baxter, Trans. Faraday Soc. 40, 546
(1944)

[29] R.E. Johnson and R.H. Dettre, J. Phys. Chem. 68, 1744 (1964).

[30] A. Dupuis and J.M. Yeomans, Langmuir 21, 2624 (2005).

[31] M. Suzuki, Carbon 32, 577 (1994)

[32] T.J. Barton et al, Chem. Mater. 11, 2633 (1999)

[33] J. Bico, C. Marzolin, D. Quere, Europhys. Lett. 47, 220 (1999)

[34] G. Carbone, L. Mangialardi, Eur. Phys. J. E. 16 67, (2005)

[35] The surface energy of a liquid does, in fact, also depend on the magnification because of thermally excited capillary waves which contribute to the surface energy of a liquid. However, the dependence of $\gamma_{1 \mathrm{v}}(\zeta)$ on the magnification $\zeta$ is rather weak and we will neglect this effect in the present study.

[36] Here we assume that the surface free energy per unit area does not depend on the orientation of the substrate surface, which may be a reasonable approximation for many amorphous materials, but which in general fails for crystalline materials.

[37] B.N.J. Persson, Eur. Phys. J. E 8, 385 (2002)

[38] S. Herminghaus, Europhys. Lett. 52 165, 2000

[39] A. Lafuma, D. Quere, Nature Mat. 2, 457 (2003)

[40] X. Gao and L. Jiang, Nature 432, 36 (2004). See also: R.B. Suter, G.E. Stratton and P.R. Miller, The Journal of Arachnology 32, 11 (2004).

[41] C. Yang, U. Tartaglino, B.N.J. Persson, Eur. Phys. J. E. 19, 47 (2006)

[42] V.N. Samoilov and B.N.J. Persson, J. Chem. Phys. 120, 1997 (2004)

[43] W.I. Jorgensen, J.D. Madura, C.J. Swenson, J. Am. Chem. Soc. 106, 6638 (1984)

[44] D.K. Dysthe, A.H. Fuchs and B. Rousseau, J. Chem. Phy. 112, $7581(2000)$

[45] U. Tartaglino, I.M. Sivebaek, B.N.J. Persson and E. Tosatti, J. Chem. Phys. 125, 014704 (2006)

[46] B.N.J. Persson, O. Albohl, U. Tartaglino, V.I. Volokitin, E. Tosatti, J. Phys. Condens. Matter 17, R1 (2005)

[47] C. Yang and B.N.J. Persson, Phys. Rev. Lett. 100, $024303(2008)$

[48] B.N.J. Persson and E. Tosatti, J. Chem. Phys. 115, 5597 (2001) 